\title{
Re: Acupuncture for recurrent urinary tract infection in women: A systematic review and meta-analysis. (First comment on BJOG-20-0142.R1)
}

\author{
Terje Alræk ${ }^{1}$ \\ ${ }^{1}$ Kristiania University College
}

June 8, 2020

\section{Dear Editor}

I read with interest the paper by Xindong Qin et al. "Acupuncture for Recurrent Urinary Tract Infection in Women: A Systematic Review and Meta-Analysis. ${ }^{1}$ In this paper, under the heading "Interpretation" they discuss possible mechanisms behind a potential effect of acupuncture. They are referring to one of my studies, ${ }^{2}$ but they are not discussing one of our study's main finding. We found a correlation between fewer urinary tract infections and less measured volume of residual urine in the women treated with acupuncture. This change in residual urine did not occurred in the non-treated group. Residual urine or post voided volume measured by a Bladder Scan, and by a nurse, in a hospital setting, and she was blinded with regard to the participants group allocation. What is an empty Bladder? A paper by Haylen discuss this and a post-voided volume above $30 \mathrm{ml}$, in otherwise healthy women, has been regarded as one of many potential risk factors ${ }^{3}$. Interestingly all women in our study had at baseline more than $30 \mathrm{ml}$ of residual urine. ${ }^{2}$ After 6 months control this was reduced to $18.2 \mathrm{~mL}$ in the acupuncture group while no change in the non-treated group ( 35.5 vs $38.8 \mathrm{~mL}$ ). Further, residual urine is recognized, and discussed as one of several potential risk factors for recurrent urinary tract infections in children ${ }^{4}$ and healthy postmenopausal women. ${ }^{5}$ Hence, with regard to post-voided volumes, I hope this can be included in future studies on acupuncture as a prophylactic treatment for recurrent urinary tract infections. Finally, a question to the authors, ${ }^{1}$ on page 6 , you write: "None of the studies reported the secondary outcomes of urinary bacteria culture, WBCs of urine dipstick, kidney function, markers of kidney damage, health-related quality of life or healthcare costs." However, our study ${ }^{2}$ used a dipstick (Uricult) and we presented the number of infections with or without bacteriuria. Do I misunderstand, or do you mean the actual name of the bacteria found?

Terje Alræk

School of Health Sciences / NAFKAM, Department of Community Medicine,

Kristiania University College / Faculty of Health Science, UiT The Arctic University of Norway

References

1. Qin X, Coyle ME, Yang L, Liang J, Wang K, Guo X, Zhang AL, Mao W, Lu C, Xue CC, Liu X. Acupuncture for recurrent urinary tract infection in women: a systematic review and meta-analysis. BJOG 2020; https://doi-org.pva.uib.no/10.1111/1471-0528.16315

2. Alraek T, Soedal LI, Fagerheim SU, Digranes A, Baerheim A. Acupuncture treatment in the prevention of uncomplicated recurrent lower urinary tract infections in adult women. Am J Public Health 2002;92:1609-11 
3. Haylen BT. The empty Bladder. Int Urogynecol J Pelvic Floor Dysfunct. 2007 Mar;18(3):237-9. doi: 10.1007/s00192-006-0111-0

4. Hoebeke P, Van Laecke E, Van Camp C, Raes A, Van De Walle J. One thousand video-urodynamic studies in children with non-neurogenic bladder sphincter dysfunction. BJU International (2001), 87, $575-580$

5. Stamm WE, Raz R. Factors contributing to susceptibility of postmenopausal women to recurrent urinary tract infections. Clin Infect Dis. 1999 Apr;28(4):723-5. doi: 10.1086/515209. 